\title{
Lactic Acidosis in a Congenital Bone Marrow Failure Syndrome
}

\author{
Fatima Farid Mir ${ }^{\mathrm{a}}$ Anjan Madasu $^{\mathrm{b}}$ Hani Humad $^{\mathrm{b}}$ Asim Noor Rana ${ }^{\mathrm{b}}$ \\ ${ }^{a}$ General Pediatrics, Dubai Health Authority, Dubai, United Arab Emirates; ${ }^{b}$ Pediatric Hematology and Oncology \\ Department, Dubai Hospital, Dubai, United Arab Emirates
}

\section{Keywords}

Pearson syndrome $\cdot$ Lactic acidosis · Mitochondrial DNA

deletion · Pancytopenia - Metabolic decompensation

\begin{abstract}
Fifteen-month-old male child, known to have a congenital bone marrow failure syndrome, presented in a state of shock with severe lactic acidosis following a brief episode of vomiting. Hospital stay was complicated by recurrent bouts of metabolic acidosis and progressive hepatic failure. Blood mitochondrial DNA sequencing revealed a large heteroplasmic 4,977 bp mitochondrial deletion (approximately $40 \%$ of all mitochondrial copies) suggestive of Pearson marrow-pancreas syndrome. By virtue of natural disease course, within a month of admission child succumbed to end-stage liver failure with multi-organ failure and died.
\end{abstract}

(c) 2021 The Author(s)

Published by S. Karger AG, Basel

\section{Introduction}

Pearson marrow-pancreas syndrome is a well-recognized entity under the category of mitochondrial deletion syndromes. Clasically, this condition has been described as a severe-onset transfusion-dependent macrocytic, sideroblastic anemia with variable degrees of neutropenia and thrombocytopenia, alongside bone marrow vacuol- ization of hematopoietic progenitor cells, hemosiderosis, and ringed sideroblasts [1]. Several reports have detailed the multi-organ reach of mitochondrial dysfunction in such patients: growth failure; cardiac impairment; neurologic regression; renal tubular dysfunction; endocrinopathies; pancreatic exocrine failure; and liver compromise are all potential syndrome components [2-7]. Probands are classically simplex cases although germline transmission has been reported [8]. Interestingly, disease phenotype has shown significant clinical overlap with other prominent mitochondrial deletion syndromes such as Kearns-Sayre syndrome [9-11]. The underlying diagnosis of Pearson marrow-pancreas syndrome is consolidated by pathognomonic bone marrow features, as well as detection of large mitochondrial DNA deletions via mitochondrial DNA analysis. Supportive evidence via metabolic work-up includes elevated plasma lactate/pyruvate and ketone body molar ratios $[2,12]$.

\section{Case Report/Case Presentation}

We present a 15-month-old male child, second in birth order to consanguineous healthy parents, delivered at term via emergency caesarian section in view of fetal distress. Antenatal course was uneventful. Within the first day of life, the infant suffered high-output cardiac failure secondary to severe anemia accompanied by thrombocytopenia. Investigations for common etiologies of neonatal bicytopenia and heart failure including TORCH karger@karger.com www.karger.com/dmj

Karger $\stackrel{\text { ' }}{=}$
(C) 2021 The Author(s)

Published by S. Karger AG, Basel

This is an Open Access article licensed under the Creative Common Attribution-NonCommercial-4.0 International License (CC BY-NC) (http://www.karger.com/Services/OpenAccessLicense), applicable to the online version of the article only. Usage and distribution for commercial purposes requires written permission.
Correspondence to:

Fatima Farid Mir, fatimafmir@gmail.com 
screening, karyotyping, echocardiography, blood film, and brain ultrasound were performed but gave no leads. Blood transfusions were required for initial stabilization, and then he was continued to be transfusion dependent requiring 4-weekly packed cell transfusion along with iron chelation. Serial blood counts continued to show bicytopenia. His growth progressed steadily around the fifteenth centile, and he was achieving normal milestones for age. The child was followed monthly by pediatric haemato-oncology team on an outpatient basis from early infancy. As bone marrow aspiration in the neonatal age-group has low diagnostic yield in marrow failure syndromes, it was deferred and a blood wholeexome sequence analysis was performed with charitable aid which was normal. The child's bicytopenia persisted and at 7 months of age the parents were then strongly encouraged to proceed to bone marrow aspiration as the next step of investigation; however, they expressed preference toward repatriation for further treatment in view of financial constraints.

Inpatient admission was required at the age of 12 months with influenza B pneumonia. Investigations at the time revealed mildly raised lactic acid and persistent pancytopenia. Child was discharged home in good condition after 3 days of antimicrobial therapy.

At the age of 13 months, the child presented to the emergency room in an obtunded state, pale, grunting, and hypotensive. The capillary blood gas analysis revealed a severe metabolic acidosis with markedly elevated lactic acid levels. His mother gave a history of moderate vomiting twice on morning of presentation followed by abrupt onset of lethargy and labored breathing. Subsequent investigations revealed anemia, thrombocytopenia, and deranged liver function tests. Inflammatory markers, blood, stool, urine cultures, and respiratory viral screening were all negative (Table 1).

The child was stabilized and admitted to pediatric intensive care unit. The therapy primarily focused on correction of the metabolic acidosis. Acid base homeostasis was achieved utilizing sodium bicarbonate infusion. With the reintroduction of feeds, the child developed large volume profuse watery diarrhea episodes triggering worsening of the lactic acidosis and clinical deterioration. As this persisted over a period of 10 days, total parental nutrition was commenced to ensure he received adequate nutrition. Sodium acetate was added to the total parental nutrition to aid the control of acid base balance and SMOF-lipid was used. The coming weeks marked an unrelenting decline to end-stage liver failure, with supportive care instituted from our side to ensure child's comfort as much as possible. Sadly, a month after admission, child went into multi-organ failure and died.

Diagnostic tests during aforementioned period yielded an acylcarnitine profile remarkable for a decreased level of $\mathrm{C} 0$ and increased level of $\mathrm{C} 18: 1, \mathrm{C} 18: 2$, and $\mathrm{C} 18-\mathrm{OH}$, and a urine organic acid analysis with markedly raised lactic acid, fumarate, and ketone bodies. With the exception of liver function derangement and increased fluid requirements, no other organ systems displayed detectable evidence of disturbance: normal creatinine, amylase/lipase, fecal elastase, and ammonia levels. Echocardiography was normal and brain imaging was not deemed necessary as child's cognitive function was age appropriate. Viral studies for Hepatitis A, Epstein-Barr virus, and Cytomegalovirus were all negative. As clinical features and basic investigations were raising suspicion toward an underlying mitochondrial disorder, initial blood wholeexome sample was re-studied with the specification to look at mi- tochondrial DNA. Reports revealed a heteroplasmic 4,977-bp mitochondrial deletion, confirming the rare diagnosis of Pearson marrow-pancreas syndrome.

\section{Discussion/Conclusion}

Mitochondrial deletion syndromes represent a spectrum of overlapping phenotypes, with features heavily dependent upon degree and distribution of affected mitochondria. Deletions have been reported to occur sporadically in the vast majority of probands, with a minority resulting from maternal germline mutations and somatic mosaicism. Given the large size and prime location of deleted material on the mitochondrial genome, disease progression and deterioration to either death or debilitation is near invariable.

Pearson syndrome holds a unique identity among the various mitochondrial deletion syndromes given its hematologic component alongside systemic affliction. Macrocytic sideroblastic anemia with a hypocellular, sideroblastic bone marrow pins the diagnosis. Extensive literature outlines the presence of multi-organ involvement, including hepatic, renal, pancreatic, and metabolic dysfunction; many of which were present in our patient as well.

Although bone marrow failure may be outgrown, Pearson syndrome holds a largely progressive disease course. Cases may die in infancy due to either sepsis, metabolic decompensation, or severe anemia, while others have been shown to progress into Kearns-Sayre syndrome.

Our reported case is the first diagnosed Pearson syndrome in the United Arab Emirates, as far as our extensive literature search has supported. The presented case's biphasic disease course and subsequent diagnostic challenges provide considerable learning opportunities. In our child, the presence of a bone marrow failure syndrome in an initially thriving infant made the likelihood of an underlying mitochondrial gene deletion lower as than the more classic presentation with anemia at a few months of age.

Moreover, several reported cases enlist failure to thrive as a common component of Pearson syndrome; however, the age at onset has yet to be detailed. Our patient developed failure to thrive only with the start of his critical illness which was 12 months after steadily gaining weight between the third and fifteenth percentiles for age.

Where the definition of Pearson syndrome has classically centered on pathognomonic bone marrow features, 
Table 1. Investigations

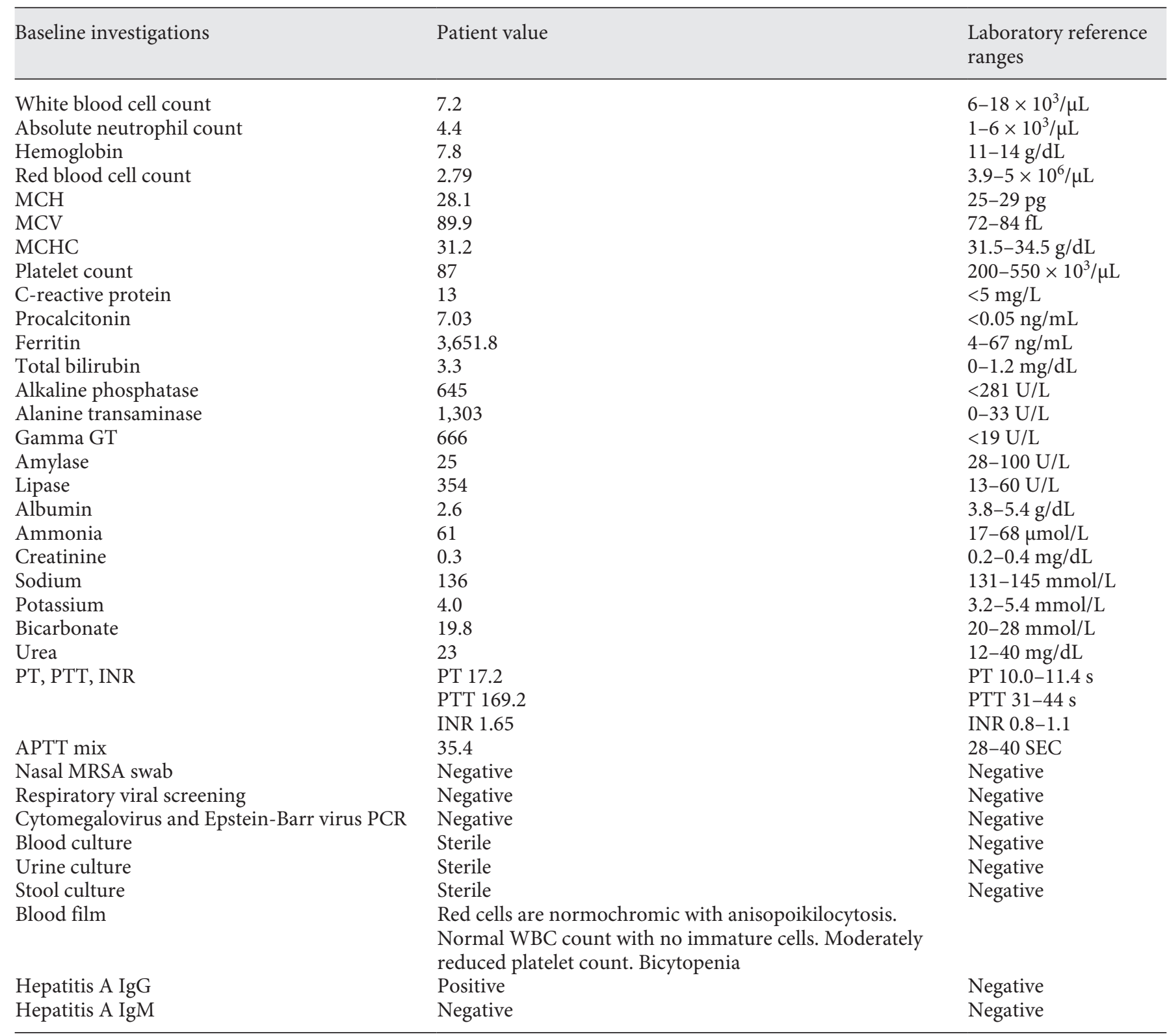

the value of performing a marrow aspirate in the neonatal age-group has recently come under question. Tadiotto et al. [13] conducted a review of 35 bone marrow analyses in genetically confirmed Pearson syndrome cases. The review confirmed that in the first month of life, $16.7 \%$ of patients with Pearson syndrome had no typical bone marrow features, while at over 1 month of age all patients presented at least one typical abnormality, with vacuolization being the most frequent feature at all ages. They noted that when a second bone marrow exam was per- formed, a pathological progression was observed, with the appearance of further alterations in almost all cases [13]. Hence, the absence of a bone marrow aspiration early in infancy, as was the case in our child due to financial constraints, or a bone marrow aspirate early in infancy without pathognomonic features are not criteria to exclude Pearson syndrome as a diagnosis.

On the other hand, blood whole-exome sequencing holds the capacity to detect mitochondrial mutations and remains the superior diagnostic modality particularly in 
infants with marrow failure, provided there has been a request to study mitochondrial DNA mutations. As elicited by our report, testing of mitochondrial gene mutations should always be part of the whole-exome sequencing request in clinically suspicious cases.

In conclusion, our reported case aims to highlight the importance of bearing the diagnosis of Pearson syndrome in mind when approaching a child with bone marrow failure and any evidence of systemic involvement. Once the disease is suspected, to concentrate on blood wholeexome sequencing with special request to search for mitochondrial genome mutations. A bone marrow analysis can be performed as a supportive modality, while considering the possibility that a bone marrow aspirate in infancy has low diagnostic yield and may not detect disease at an early age. Once the diagnosis of Pearson syndrome has been attained, care is focused on supportive and palliative care for the remainder of the child's life as no cure exists to date.

\section{Statement of Ethics}

Written informed consent was obtained from the patient's parents to write and publish this case report.

\section{Conflict of Interest Statement}

The authors have no conflicts of interest to declare.

\section{Funding Sources}

The authors did not receive any funding.

\section{Author Contributions}

All the authors have contributed to writing, editing, and finalizing the case report.

\section{References}

1 Goldstein A, Falk MJ, Adam MP, Ardinger $\mathrm{HH}$, Pagon RA, Wallace SE, et al. Mitochondrial DNA deletion syndromes. In: GeneReviews. Seattle, WA: University of Washington; 2003 Dec 17.

2 Al-Tamemi SH. Pearson's marrow-pancreas syndrome. Sultan Qaboos Univ Med J. 2009 Aug;9(2):196-7.

3 Corresponding author JF. Postfach 20. 1180 Vienna, Austria, Europe. The Letter To Editor. 2018.

4 Khasawneh R, Alsokhni H, Alzghoul B, Momani A, Abualsheikh N, Kamal N, et al. A novel mitochondrial DNA deletion in patient with pearson syndrome. Med Arch. 2018 Apr; 72(2):148-50.

5 Reddy JM, Jose J, Prakash A, Devi S. Pearson syndrome: a rare inborn error of metabolism with bone marrow morphology providing a clue to diagnosis. Sudan J Paediatr. 2019; 19(2):161-4.
6 Farruggia P, Di Cataldo A, Pinto RM, Palmisani E, Macaluso A, Valvo LL, et al. Pearson syndrome: a retrospective cohort study from the Marrow Failure Study Group of A.I.E.O.P. (Associazione Italiana Emato-Oncologia Pediatrica). JIMD Rep. 2016;26:37-43.

7 Kitano T, Yoshida S. Pearson syndrome as a rare cause of failure to thrive, anemia and exocrine pancreatic insufficiency: a case report. Pediatr Ther. 2018;8(1):341.

8 Shanske S, Tang Y, Hirano M, Nishigaki Y, Tanji K, Bonilla E, et al. Identical mitochondrial DNA deletion in a woman with ocular myopathy and in her son with pearson syndrome. Am J Hum Genet. 2002;71(3):679-83.

9 Gustafson MA, McCormick EM, Perera L, Longley MJ, Bai R, Kong J, et al. Mitochondrial single-stranded DNA binding protein novel de novo SSBP1 mutation in a child with single large-scale mtDNA deletion (SLSMD) clinically manifesting as Pearson, Kearns-Sayre, and Leigh syndromes. PLoS One. 2019;14(9):e0221829.
10 Arzanian MT, Eghbali A, Karimzade P, Ahmadi M, Houshmand M, Rezaei N. mtDNA deletion in an Iranian infant with Pearson Marrow syndrome. Iran J Pediatr. 2009 Jul 6; 20(1):107-12.

11 McShane MA, Hammans SR, Sweeney M, Holt IJ, Beattie TJ, Brett EM, et al. Pearson syndrome and mitochondrial encephalomyopathy in a patient with a deletion of mtDNA. Am J Hum Genet. 1991 Jan;48(1):39-42.

12 Park J, Ryu H, Jang W, Chae H, Kim M, Kim $\mathrm{Y}$, et al. Novel $5.712 \mathrm{~kb}$ mitochondrial DNA deletion in a patient with Pearson syndrome: a case report. Mol Med Rep. 2015 May;11(5): 3741-5.

13 Tadiotto E, Maines E, Degani D, Balter R, Bordugo A, Cesaro S. Bone marrow features in Pearson syndrome with neonatal onset: a case report and review of the literature. Pediatr Blood Cancer. 2018 Apr;65(4):e26939. 